\title{
Influence of Cold Rolling on Diffusion Bondability of SUS316L Stainless Steel Sheets
}

\author{
Takashi Harumoto, Yuya Yamashita, Osamu Ohashi and Takashi Ishiguro* \\ Department of Materials Science and Technology, Faculty of Industrial Science and Technology, \\ Tokyo University of Science, Tokyo 125-8585, Japan
}

The influence of cold rolling on diffusion bondability has been systematically investigated using SUS316L stainless steel sheets with different reduction ratios $(0,35,50$ and $70 \%)$ as specimens. According to the results of tensile testing of diffusion bonds, highly rolled sheets exhibit enhanced diffusion bondability. This phenomenon is discussed from the viewpoint of the microstructure. [doi:10.2320/matertrans.M2013405]

(Received November 5, 2013; Accepted December 25, 2013; Published February 15, 2014)

Keywords: diffusion bonding, cold rolled sheets, 316L, stainless steel, reduction

\section{Introduction}

As the diffusion bonding method allows accurate fabrication, it has been applied to manufacture reactors from metallic sheets, such as reactors for the nuclear industry and micro-reactors for heat exchange. ${ }^{1-4)}$ Multilayered steel sheets prepared using the diffusion bonding method have also been investigated and anomalous large elongation was reported. ${ }^{5-7)}$

Since the diffusion bonding method is based on the diffusion of atoms, diffusion bondability is influenced by the microstructure and surface conditions, which depend on the process of specimen fabrication and especially final treatment of bonding surfaces. ${ }^{8-15}$ ) For example, enhanced diffusion bondability is achieved with fine-grained structures. Usually, these investigations are conducted on cylinder/cylinder and block/block or sheet/sheet under shear configuration, as their mechanical properties can readily be measured. In contrast, tensile strength of sheet/sheet has been investigated less extensively, as the tensile testing of such bonds is difficult. ${ }^{16,17)}$ Especially, tensile testing of sheet/sheet bonds along the sheet normal direction (ND) has not been performed. However, it is important to investigate the tensile strength of sheet/sheet along ND, as reactors with complicated structures are fabricated by stacking sheets.

As the microstructure of sheets can be markedly changed by final treatment, such as cold rolling, ${ }^{18)}$ it is necessary to study the diffusion bondability of cold rolled sheets. Indeed, the bonding temperature of sheets is known to vary between lots and something unknown parameters.

In this study, we investigated the tensile strength of sheet/ sheet diffusion bonds along ND and the influence of cold rolling on diffusion bondability. Diffusion bonds with different cold rolling reduction ratios were prepared, and the influence of cold rolling on diffusion bondability of SUS316L stainless steel sheets was systematically investigated from the viewpoint of microstructure.

*Corresponding author, E-mail: ishiguro@ @rs.noda.tus.ac.jp
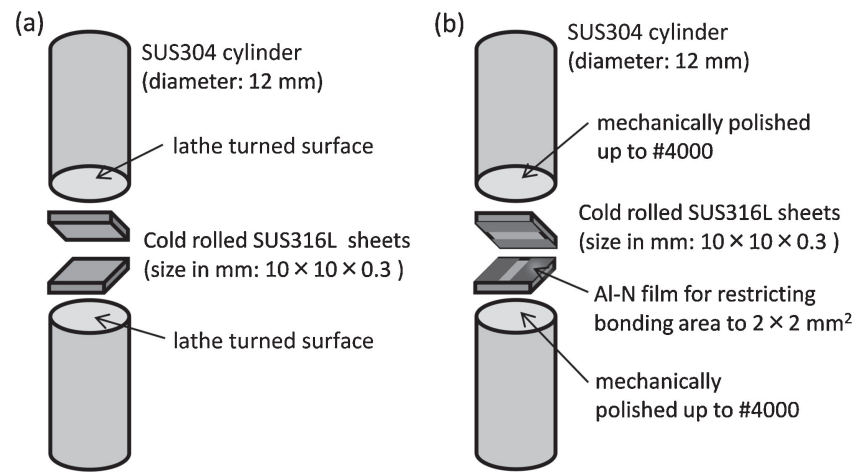

Fig. 1 Fabricated diffusion bonds of (a) type-A and (b) type-B.

\section{Experimental Procedures}

To fabricate SUS316L stainless steel sheets with different reduction ratios, annealed SUS316L sheets (thickness: 462, 600 , and $1000 \mu \mathrm{m}$ ) were cold rolled to $300 \mu \mathrm{m}$ (cold rolled sheets were supplied by WELCON Co. Ltd., Niigata, Japan). Thus, the reduction ratios were 35,50 , and $70 \%$, respectively. Annealed sheet with a thickness of $300 \mu \mathrm{m}$ were adopted as $0 \%$ cold rolled sheets. The prepared sheets were cut into $10 \mathrm{~mm}$ squares.

Two types of diffusion bonds were prepared (Fig. 1). As one diffusion bond, two SUS316L sheets were simply coupled and then compressed by SUS304 cylinders as shown in Fig. 1(a) (hereafter, this type of bond is described as typeA). The diameter of the cylinders was $12 \mathrm{~mm}$ and the bonding surface was turned by lathe. In type-B bonds, two bonding conditions, bonding area and surface treatment of the cylinders, were modified. To restrict the bonding area to $4 \mathrm{~mm}^{2}$, partially nitrided aluminum (Al-N) film $300 \mathrm{~nm}$ thick was sputter-deposited onto $10 \mathrm{~mm}$ square SUS316L sheets as shown in Fig. 1(b). The details of deposition conditions of the film were described in our previous reports. ${ }^{19,20)}$ As the sheets with Al-N were stacked on each other in cross direction, the bonding area was $2 \times 2 \mathrm{~mm}^{2}$. The bonding surfaces of the cylinders of SUS304 were polished up to \#4000. 
Diffusion bonding of the two types of bonds was conducted using a high-frequency induction heating apparatus (FIH-702; Fuji Electric Industrial Co. Ltd., Saitama, Japan). The sheets and cylinders were rinsed using acetone and $100 \mathrm{kgf}$ was applied via cylinders, which is equivalent to $9.8 \mathrm{MPa}$ at the sheet/sheet interface. The temperature was increased at a rate of $250 \mathrm{~K} / \mathrm{min}$, and kept at $1073-1273 \mathrm{~K}$ for $30 \mathrm{~min}$ for diffusion bonding. The specimen was then cooled to room temperature. All processes of heating, holding, and cooling were performed in a vacuum to prevent oxidation. The pressure before heating was better than $3.0 \times 10^{-4} \mathrm{~Pa}$.

The mechanical properties of the prepared diffusion bonds were measured using a tensile testing machine at room temperature (AG-10TA; Shimadzu Co. Ltd., Kyoto, Japan). The strain rate was controlled as $0.05 \mathrm{~mm} / \mathrm{min}$. The load was converted to stress using the bonding area $\left(100 \mathrm{~mm}^{2}\right.$ for typeA and $4 \mathrm{~mm}^{2}$ for type-B). The fracture surface and crosssection of the bonds were observed by scanning electron microscopy (SEM) (JSM-5800LV; JEOL Co. Ltd., Tokyo, Japan) and optical microscopy (VHX-500; Keyence Co. Ltd., Osaka, Japan). Cross-sections of specimens were mechanically polished and finally etched with $\mathrm{FeCl}_{2}$ aqueous solution.

To investigate the recrystallization behaviors of cold rolled SUS316L sheets, the sheets were annealed in a vacuum at $773-1273 \mathrm{~K}$ and their Vickers hardness were measured (MVK-EII; Akashi Co. Ltd., Tokyo, Japan). The annealed sheets were also analyzed by X-ray diffractometer with a rotating copper anode (XRD) (RU-300; Rigaku Co. Ltd., Tokyo, Japan).

\section{Results and Discussion}

\subsection{Tensile testing}

The fabricated type-A and type-B diffusion bonds with different reduction ratios were subjected to tensile testing without machining. As type-A bonds fractured at the sheet/ cylinder interface, it was impossible to measure the tensile strength of sheet/sheet bonding. According to cross-sectional observations, the roughened surface of the cylinders results in poor tensile strength of the sheet/cylinder interface. Thus, the bonding surface of the cylinders should be polished and the bonding area of the sheet/cylinder should be larger than that of the sheet/sheet.

These problems were solved using type-B bonds in which the tensile fracture occurred at the sheet/sheet boundary. Therefore, the tensile strength of the SUS316L sheets with different reduction ratios could be measured properly. Figure 2 shows the bonding temperature dependence of the tensile strength of type-B diffusion bonds. Highly cold rolled sheets, such as $70 \%$ sheets, could be bonded at lower temperature. Thus, diffusion bondability is enhanced by cold rolling.

\subsection{Microstructure of diffusion bonds}

To determine the details of the relation between reduction ratio and diffusion bondability, fracture surface observation, cross-sectional observation, and Vickers hardness measurement were performed.

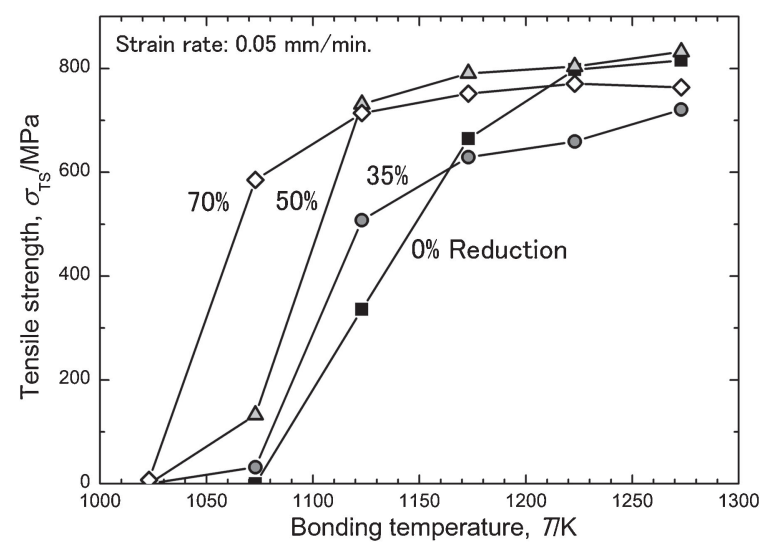

Fig. 2 Tensile strength of type-B diffusion bonds.

SEM images of fracture surfaces of diffusion bonds are shown in Fig. 3. It can be clearly seen that the fracture surface is roughened and dimples are increased at higher reduction ratios and higher bonding temperatures. It was also confirmed that the bonding area is increased at higher reduction ratios and higher bonding temperatures. Thus, the improvement in the bonding strength at the bonding area and the increase in bonding area result in enhanced tensile strength of diffusion bonds.

Figure 4 shows cross-sectional optical images of the sheet/sheet interface. As shown in these images, recrystallization of 50 and $70 \%$ reduction sheets takes place and is almost complete at $1173 \mathrm{~K}$, and some of the grains grow over the bonding interface at $1273 \mathrm{~K}$. It should be noted that the bonding boundary of $70 \%$ sheets is fading out. In contrast, recrystallization of $35 \%$ reduction sheets is still in progress at $1173 \mathrm{~K}$ and is not complete at $1273 \mathrm{~K}$.

Vickers hardness measurement is also adopted to investigate recrystallization and grain growth behaviors of the sheets with different reduction ratios (Fig. 5). The results of tensile testing are also shown in this figure. Cold rolled sheets were annealed for $30 \mathrm{~min}$, which is the same duration as diffusion bonding, and softening by annealing is observed. The $70 \%$ cold rolled sheets exhibit softening at lower temperatures compared with 50 and $30 \%$ cold rolled sheets. The softening of $70 \%$ sheets is also confirmed from the stress-strain curve as a decrease in Young's modulus. This softening is related to the tensile strength of the diffusion bonds: the rapid increase in tensile strength is observed at the temperature at which softening is completed. Thus, the diffusion bonding could be mainly dominated by volume diffusion rather than grain boundary diffusion.

XRD profiles of cold rolled sheets are shown in Fig. 6. Detected peaks are related to austenite $(\gamma)$, except for a strain-induced martensite $\left(\alpha^{\prime}\right)(211)$ peak. However, the effect of $\alpha^{\prime}$ on diffusion bondability can be excluded for two reasons as follows. (i) As the peak intensity of $\alpha^{\prime}$ (211) peak is very weak compared with $\gamma$ peaks, the amount of $\alpha^{\prime}$ is very small. (ii) Although enhanced bondability during phase transformation and dynamic recrystallization in duplex stainless steel has been reported, ${ }^{21-23)}$ transformation from $\alpha^{\prime}$ to $\gamma$ is completed at the bonding temperature. ${ }^{24-26)}$ Thus, $\alpha^{\prime}$ phase cannot influence the diffusion bondability at 1000 $1200 \mathrm{~K}$. 


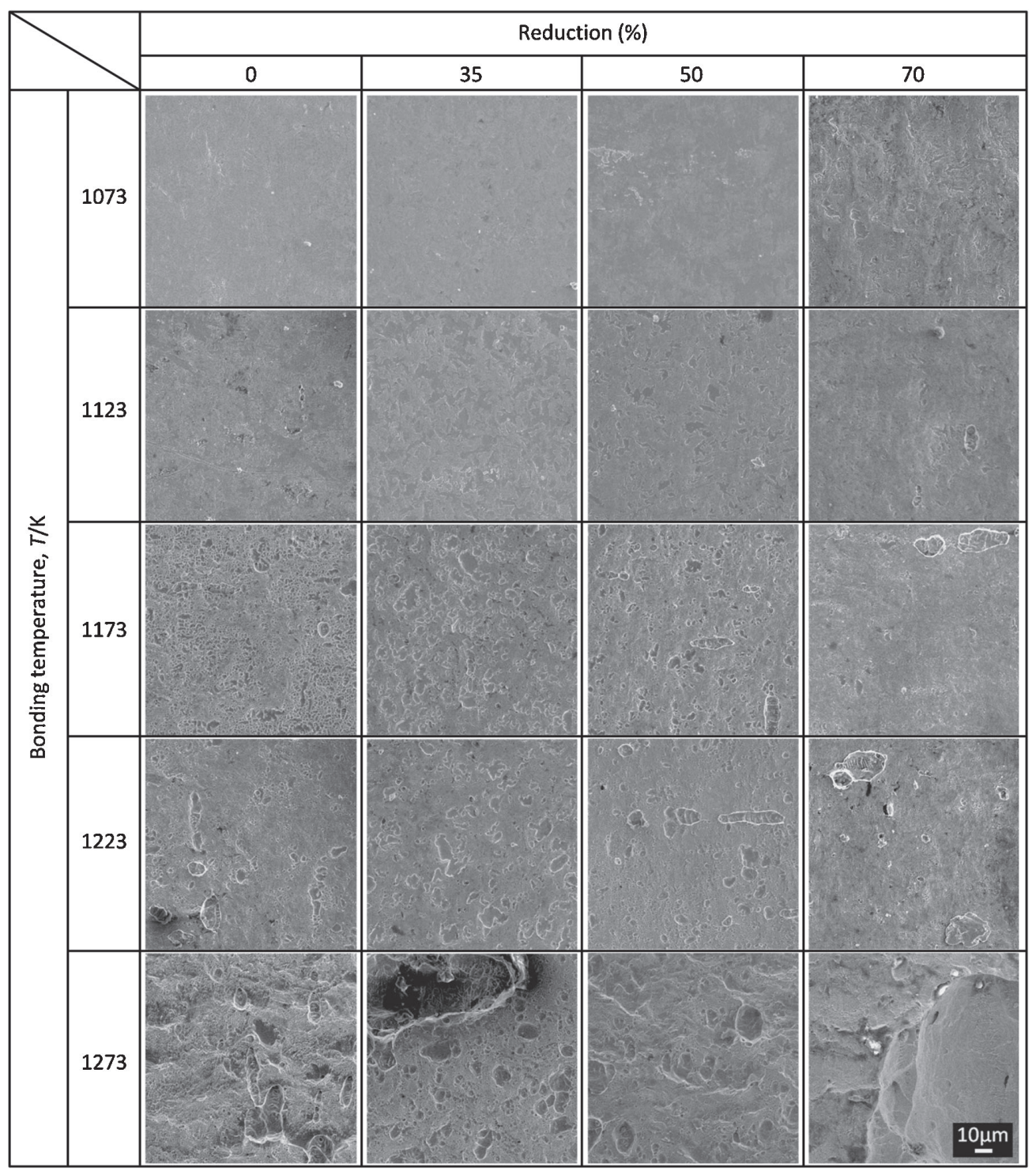

Fig. 3 SEM images of fracture surfaces of type-B diffusion bonds.

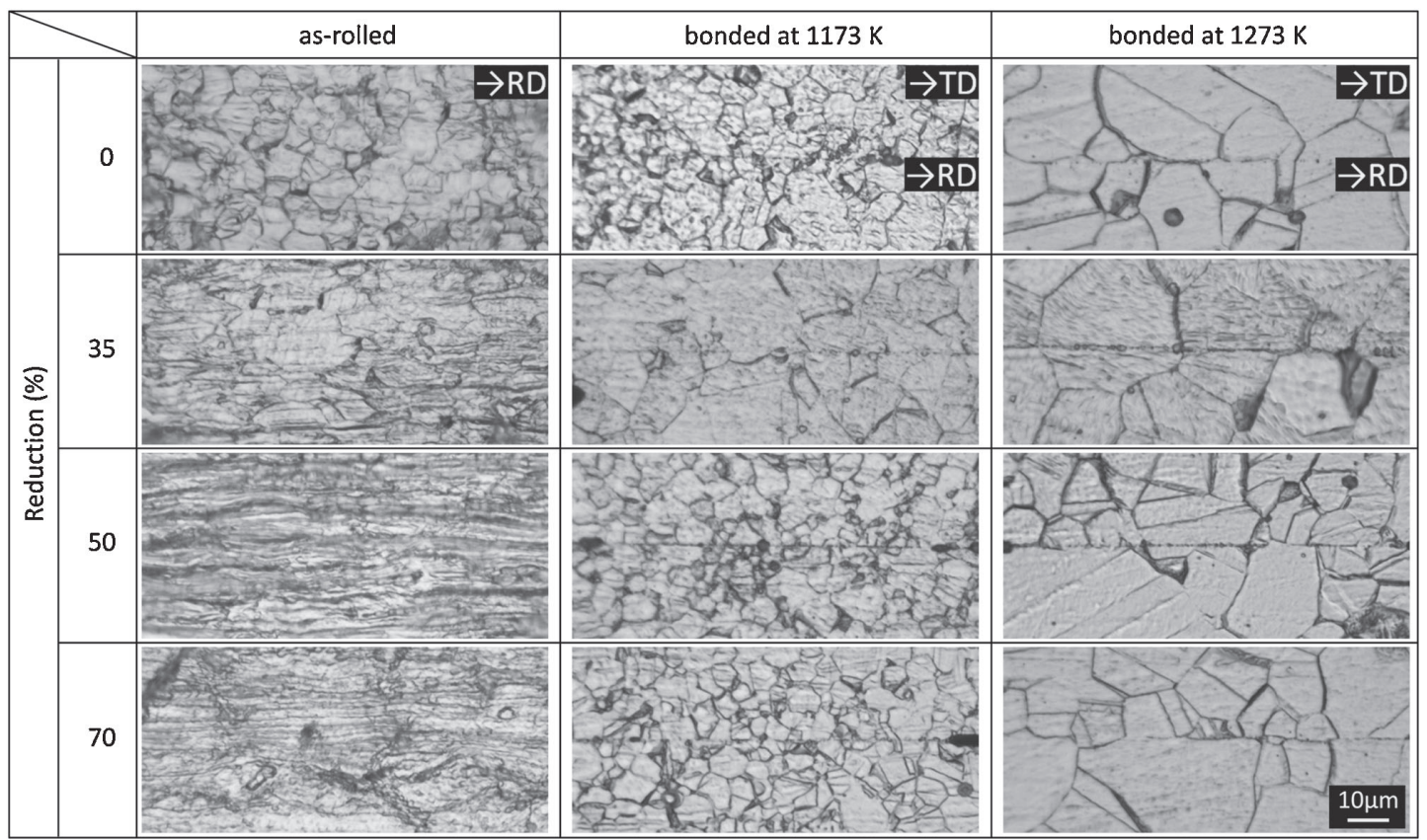

Fig. 4 Cross-sectional optical micrographs of type-B diffusion bonds. 


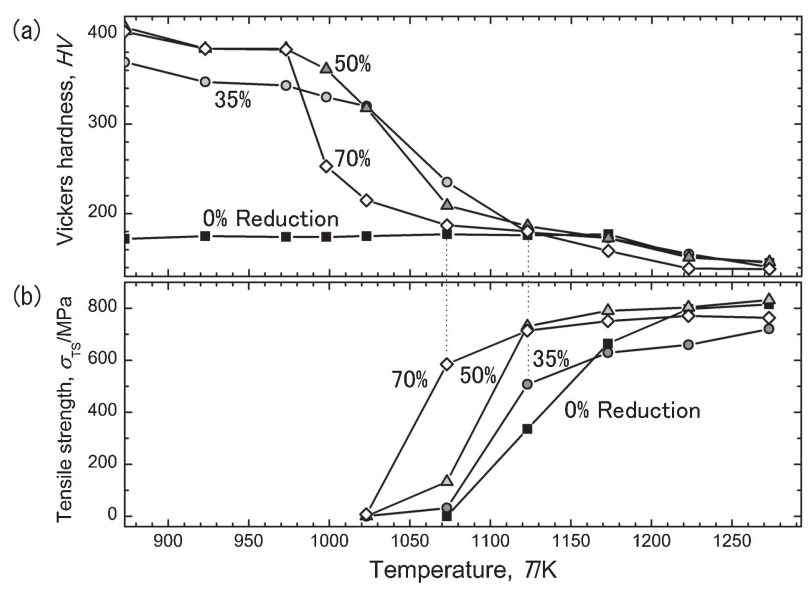

Fig. 5 (a) Vickers hardness of annealed sheets and (b) tensile strength of type-B diffusion bonds

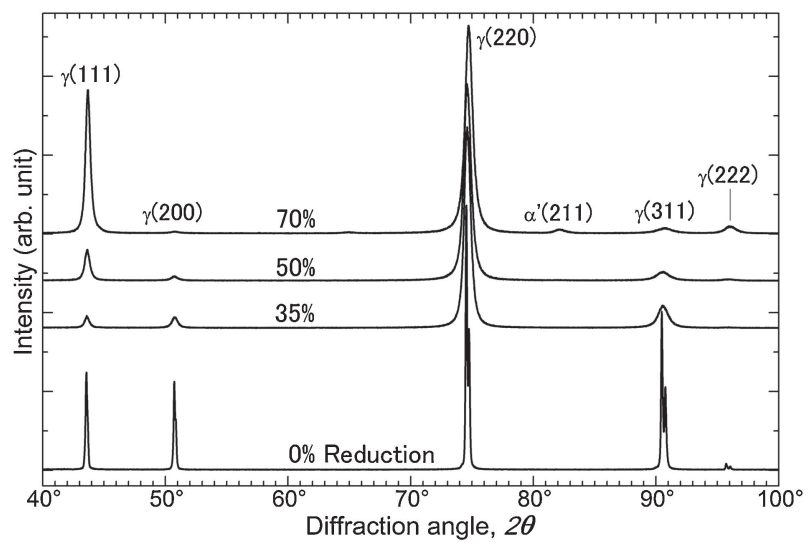

Fig. 6 XRD profiles of cold rolled SUS316L sheets. $\gamma$ and $\alpha^{\prime}$ are correspond to austenite and strain-induced martensite, respectively.

\section{Conclusion}

Diffusion bondability of cold rolled SUS316L sheets has been investigated. The results of tensile testing of the fabricated present novel diffusion bonds indicated that highly cold rolled sheets exhibit enhanced diffusion bondability. This phenomenon can be explained by the low temperature recrystallization and the high volume diffusion of highly cold rolled sheets, resulting in improvement in bonding strength at bonding area and increase in bonding area. It should be noted that although only SUS316L sheets were examined in this study, this conclusion can be applied to other austenitic stainless steel.

\section{Acknowledgments}

Cold rolled SUS316L sheets were supplied by WELCON Co. Ltd., Niigata, Japan.

\section{REFERENCES}

1) K. Bhanumurthy and G. B. Kale: J. Mater. Sci. Lett. 12 (1993) 18791881.

2) T. Takeda, K. Kunitomi and T. Horie: Nucl. Eng. Des. 168 (1997) 11-21.

3) S. X. Li, L. Li, S. R. Yu, R. Akid and H. B. Xia: Corros. Sci. 53 (2011) 99-104.

4) Y. Suzuki, T. Saito and Y. Abe: J. Jpn. Soc. Technol. 52 (2011) 434438.

5) S. Nambu, M. Michiuchi, Y. Ishimoto, K. Asakura, J. Inoue and T. Koseki: Scr. Mater. 60 (2009) 221-224.

6) S. Nambu, M. Michiuchi, J. Inoue and T. Koseki: Compos. Sci. Technol. 69 (2009) 1936-1941.

7) A. Çetin, C. Bernardi and A. Mortensen: Acta Mater. 60 (2012) 22652276.

8) I. Masumoto and H. Hira: J. Jpn. Weld. Soc. 5 (1981) 68-74.

9) A. S. Zuruzi, H. Li and G. Dong: Mater. Sci. Eng. A 270 (1999) 244 248.

10) A. Wang, O. Ohashi, M. Aoki and N. Yamaguchi: ISIJ Int. 42 (2002) 1386-1390.

11) H. Somekawa, H. Watanabe and K. Higashi: Mater. Trans. 44 (2003) 496-503.

12) S. D. Chen, F. J. Ke, M. Zhou and Y. L. Bai: Acta Mater. 55 (2007) 3169-3175.

13) M. Joseph Fernandus, T. Senthilkumar, V. Balasubramanian and S. Rajakumar: J. Mater. Eng. Perform. 21 (2012) 2303-2315.

14) H. Y. Chen, J. Cao, X. Y. Tian, R. Li and J. C. Feng: Appl. Phys. A 113 (2013) 101-104.

15) J. Cao, H. Y. Chen, X. G. Song, J. K. Liu and J. C. Feng: J. Non-Cryst. Solids 364 (2013) 53-56.

16) S. X. Li, F. Z. Xuan and S. T. Tu: J. Nucl. Mater. 366 (2007) 1-7.

17) S. X. Li, F. Z. Xuan, S. T. Tu and S. R. Yu: Mater. Sci. Eng. A 491 (2008) 488-491.

18) N. Hirota, F. X. Yin, T. Inoue and T. Azuma: ISIJ Int. 48 (2008) 475482.

19) T. Ishiguro, M. Nishimura and T. Yamazaki: Jpn. J. Appl. Phys. 41 (2002) 292-300.

20) T. Ishiguro and H. Iijima: Jpn. J. Appl. Phys. 45 (2006) 7784-7788.

21) O. Ohashi and T. Hashimoto: J. Jpn. Weld. Soc. 49 (1980) 24-29.

22) K. Tsuzaki, H. Matsuyama, M. Nagao and T. Maki: J. Japan Inst. Metals 54 (1990) 878-887.

23) M. Sakamoto, T. Tsuzuki, H. Moriai, T. Kobayashi and K. Kusaka: Mitsubishi Heavy Ind. Tech. Rev. 37 (2000) 134-137.

24) S. Takaki: Tetsu-to-Hagane 80 (1994) N529-N535.

25) A. F. Padilha, R. L. Plaut and P. R. Rios: ISIJ Int. 43 (2003) 135-143.

26) M. Katoh and Y. Torisaka: Tetsu-to-Hagane 93 (2007) 23-26. 